\title{
AN EMBEDDING THEOREM WITH AMALGAMATION FOR CANCELLATIVE SEMIGROUPS $\dagger$
}

\author{
by J. M. HOWIE
}

(Received 5 February, 1962)

1. Introduction. Let $\left\{S_{i} ; i \in I\right\}$ be a finite or infinite family of cancellative semigroups. Let $U$ be a cancellative semigroup, and suppose that there exists, for each $i$ in $I$, a monomorphism $\phi_{i}: U \rightarrow S_{i}$. We are interested in finding a semigroup $T$ with the following properties.

(a) For each $i$ in $I$, there is a monomorphism $\lambda_{i}: S_{i} \rightarrow T$ such that $u \phi_{i} \lambda_{i}=u \phi_{j} \lambda_{j}$ for all $u \in U$ and all $i, j$ in $I$. That is to say, there exists a monomorphism $\lambda: U \rightarrow T$ which equals $\phi_{i} \lambda_{i}$ for all $i$ in $I$.

(b) $S_{i} \lambda_{i} \cap S_{j} \lambda_{j}=U \lambda \quad(i, j \in I ; i \neq j)$.

More briefly, we wish to find a semigroup $T$ containing isomorphic copies of each of the $S_{i}$, intersecting in an isomorphic copy of $U$. When such a $T$ can be found, we shall say that the embedding $\left[\left(S_{i}\right) ; U\right]$ is possible. If moreover we can find a $T$ which is cancellative, we shall say that the cancellative embedding $\left[\left(S_{i}\right) ; U\right]$ is possible.

In [4], the author considered the corresponding problem for a family $\left(S_{i}\right)$ of (not necessarily cancellative) semigroups, and showed that the embedding $\left[\left(S_{i}\right) ; U\right]$ was possible if $U$ was a group. In this paper we show that if $U$ is a group, then the cancellative embedding $\left[\left(S_{i}\right) ; U\right]$ is also possible.

DEFINITION (Dubreil [2]). A subsemigroup $U$ of a semigroup $S$ is said to be unitary in $S$ if, for all $u \in U, s \in S$,

(i) $u s \in U \Rightarrow s \in U$; and (ii) $s u \in U \Rightarrow s \in U$.

If, for each $i$ in $I$, the subsemigroup $U \phi_{i}$ is unitary in $S_{i}$, then the embedding $\left[\left(S_{i}\right) ; U\right]$ is possible [4, Corollary 3.4]. In the final section of this paper we show, by means of an example, that the cancellative embedding $\left[\left(S_{i}\right) ; U\right]$ may be impossible.

2. The group case. We prove the following theorem.

THEOREM 2.1. Let $\left\{S_{i} ; i \in I\right\}$ be a family of cancellative semigroups, and suppose that there exists a group $U$ and a monomorphism $\phi_{i}: U \rightarrow S_{i}$ for each $i$ in $I$. Then the cancellative embedding $\left[\left(S_{i}\right) ; U\right]$ is possible.

In the proof we shall use the following notation. Elements of $U$ will be denoted by symbols such as $u, u^{\prime}, u^{(n)}$, etc. For any $i$ in $I$, the symbols $u_{i}$, $u_{i}^{\prime}$, and $u_{i}^{(n)}$ will then mean $u \phi_{i}$, $u^{\prime} \phi_{i}$, and $u^{(n)} \phi_{i}$ respectively. We shall similarly use $v, v^{\prime}, v^{(n)}$, etc. for elements of $U$.

The method of approach to the proof of this theorem is to consider, as in [4], the free product $\Pi_{i}^{*} S_{i}$ of the semigroups $S_{i}$, amalgamating $U$. The definition of the amalgamated free product that is most convenient for the purposes of the present paper is as follows.

$\uparrow$ The results of $\$ 2$ were contained in a thesis submitted to Oxford University for the degree of D.Phil. I am grateful to Professor G. Higman and Dr G. B. Preston for much valuable advice, and also to D.S.I.R. for financial support. 
First, consider the set $W$ of finite, non-empty words $a_{1} a_{2} \ldots a_{m}$, with each $a_{r}(r=1,2, \ldots, m)$ an element of some $S_{i}$. If multiplication in $W$ is defined by juxtaposition, it is clear that $W$ becomes a semigroup. Now define elementary equivalences on the elements of $W$ as follows.

(E1) If, in the element $a_{1} a_{2} \ldots a_{m}$ of $W$, two adjacent letters $a_{r}$ and $a_{r+1}$ are in the same $S_{i}$, and if $a_{r} a_{r+1}=a_{r}^{*}$ in $S_{i}$, then $a_{1} a_{2} \ldots a_{m}$ and $a_{1} a_{2} \ldots a_{r-1} a_{r}^{*} a_{r+2} \ldots a_{m}$ are said to be elementarily equivalent to each other.

(E2) If, in the element $a_{1} a_{2} \ldots a_{m}$ of $W$, some $a_{r}=u_{i} \in U \phi_{i}$, and if $a_{r}^{*}$ is defined to be $u_{j}$ $\left(=u_{i} \phi_{l}^{-1} \phi_{j}\right)$, where $j$ is any element of $I$, then $a_{1} a_{2} \ldots a_{m}$ and $a_{1} a_{2} \ldots a_{r-1} a_{r}^{*} a_{r+1} \ldots a_{m}$ are said to be elementarily equivalent to each other.

Next, define two elements of $W$ as equivalent if they can be connected by a finite (perhaps empty) sequence of elementary equivalences of types (E1) and (E2). It is easy to verify that this is indeed an equivalence relation on the semigroup $W$, and in fact it is even a congruence. We shall denote this congruence by $\rho$. Now we define $\Pi_{U}^{*} S_{i}$, the free product of the semigroups $S_{i}$ amalgamating $U$, as the quotient semigroup of $W$ by the congruence $\rho$. This definition can be shown to be equivalent to the definition in [4].

Now, from [4, Corollary 3.7 and Theorem 2.3], we know that when $U$ is a group, $\Pi_{U}^{*} S_{i}$ is a semigroup with the properties $(a)$ and $(b)$ listed in $\S 1$. It is therefore clear that Theorem 2.1 will be proved if we prove the following lemma.

LEMMA 2.2. Under the conditions of Theorem $2.1, \Pi_{U}^{*} S_{i}$ is a cancellative semigroup.

To prove this, it is necessary first to make some observations regarding subgroups of cancellative semigroups.

Suppose then that a cancellative semigroup $S$ contains a subgroup $U$. Let $e$ be the identity element of $U$. Then $e$ must be an identity element for the whole of $S$, for certainly $e^{2}=e$, and therefore, for any $s$ in $S$,

$$
e^{2} s=e s, \text { and } s e^{2}=s e .
$$

By cancellation, it follows that $e s=s$ and $s e=s$ for all $s$ in $S$.

The right cosets $U s$ of $U$ in $S$ satisfy the condition

$$
U s \cap U s^{\prime} \neq \square \Rightarrow U s=U s^{\prime},
$$

for $s, s^{\prime}$ in $S$. For, since $U$ is a group, we have that $U u=U$ for all $u$ in $U$, and therefore

$$
\begin{gathered}
U s \cap U s^{\prime} \neq \square \Rightarrow \exists u, u^{\prime} \in U \text { such that } u s=u^{\prime} s^{\prime} \\
\Rightarrow U u s=U u^{\prime} s^{\prime} \Rightarrow U s=U s^{\prime} .
\end{gathered}
$$

Also, every element of $S$ is contained in some right coset of $U$, for the element $s$ is contained in the coset $U s$.

If now a representative element is chosen in each right coset of $U$, the choice being arbitrary (except that $e$ is chosen as the representative of the coset $U e$ ), but thereafter fixed, it follows that every element $s$ in $S$ has a unique representation

$$
s=u z,
$$

where $u \in U$, and $z$ is a coset representative. For, by (1), the element $s$ can belong to only one right coset of $U$, so that $z$ is uniquely determined. If now $s=u z$, and $s=u^{\prime} z$, it is immediate by cancellation that $u=u^{\prime}$. Thus $u$ also is uniquely determined. 
Finally, a point of terminology: if a coset representative $z$ is distinct from $e$, we shall call it non-trivial.

Now, returning to the proof of Lemma 2.2, we have that, for each $i$ in $I, U \phi_{i}$ is a subgroup of the cancellative semigroup $S_{i}$. The foregoing therefore applies, and we choose, for each $i$ in $I$, a system of representatives for the cosets of $U \phi_{i}$ in $S_{i}$. It is now possible to follow exactly the procedure in [3, Chapter 17] (see also [5], [6]) and to find a canonical form

$$
u z_{1} z_{2} \ldots z_{n}
$$

for elements in $\Pi_{U}^{*} S_{l}$, where $u \in U$, and $z_{1}, z_{2}, \ldots z_{n}$ are non-trivial coset representatives in the$S_{i}$ 's such that no two adjacent $z$ 's belong to the same $S_{i}$. The details in the proof of this state ment are identical with those in the group case and are omitted. Stating the result more precisely, we have that any element $a$ of $\Pi_{U}^{*} S_{i}$ is an equivalence class under $\rho$ of words in the elements of the $S_{i}$ 's, and that this class contains, for an arbitrary fixed $h$ in $I$, one and only one word of the form $u_{h} z_{1} z_{2} \ldots z_{n}$, in which the $z$ 's are non-trivial coset representatives, and no two adjacent $z$ 's are in the same $S_{i}$. Because of the arbitrariness of the $h$, it is possible to drop the suffix on the $u$, and to identify the form $u z_{1} \ldots z_{n}$ with the $\rho$-class $\left(u_{h} z_{1} \ldots z_{n}\right) \rho$.

Suppose now that, in $\Pi_{U}^{*} S_{i}$,

$$
x a=y a .
$$

We wish to show that $x=y$. Suppose that the canonical words associated with $x$ and $y$ are respectively $u_{h}^{\prime} z_{1}^{\prime} \ldots z_{m}^{\prime}$ and $u_{h}^{\prime \prime} z_{1}^{\prime \prime} \ldots z_{n}^{\prime \prime}$. The element $a$ may be written as

$$
\left(u_{h} z_{1} \ldots z_{l}\right) \rho=\left(u_{h} \rho\right)\left(z_{1} \rho\right) \ldots\left(z_{l} \rho\right)
$$

and therefore it will be sufficient to prove that

and

$$
\begin{aligned}
& \text { (i) } x\left(u_{h} \rho\right)=y\left(u_{h} \rho\right) \Rightarrow x=y, \\
& \text { (ii) } x(z \rho)=y(z \rho) \Rightarrow x=y,
\end{aligned}
$$

where $z$ is a non-trivial coset representative.

The hypothesis in (i) is that

$$
u_{h}^{\prime} z_{1}^{\prime} \ldots z_{m}^{\prime} u_{h} \equiv u_{h}^{\prime \prime} z_{1}^{\prime \prime} \ldots z_{n}^{\prime \prime} u_{h} \quad(\rho) .
$$

Since $\rho$ is a congruence, it follows that

i.e. that

$$
u_{h}^{\prime} z_{1}^{\prime} \ldots z_{m}^{\prime} u_{h} u_{h}^{-1} \equiv u_{h}^{\prime \prime} z_{1}^{\prime \prime} \ldots z_{n}^{\prime \prime} u_{h} u_{h}^{-1} \quad(\rho)
$$

where $e_{h}$ is the identity element of $U \phi_{h}$. Now suppose that $z_{m}^{\prime} \in S_{j}$. Then

$$
u_{h}^{\prime} z_{1}^{\prime} \ldots z_{m}^{\prime} e_{h} \equiv u_{h}^{\prime} z_{1}^{\prime} \ldots z_{m}^{\prime} e_{j} \equiv u_{h}^{\prime} z_{1}^{\prime} \ldots z_{m}^{\prime} \quad(\rho)
$$

for the first two words are elementarily equivalent by (E2), and the last two are elementarily equivalent by (E1). Similarly

and we conclude that

$$
\begin{gathered}
u_{h}^{\prime \prime} z_{1}^{\prime \prime} \ldots z_{n}^{\prime \prime} e_{h} \equiv u_{h}^{\prime \prime} z_{1}^{\prime \prime} \ldots z_{n}^{\prime \prime} \quad(\rho), \\
u_{h}^{\prime} z_{1}^{\prime} \ldots z_{m}^{\prime} \equiv u_{h}^{\prime \prime} z_{1}^{\prime \prime} \ldots z_{n}^{\prime \prime} \quad(\rho),
\end{gathered}
$$

i.e. that $x=y$. 
In (ii) we are considering the two words

and

$$
u_{h}^{\prime} z_{1}^{\prime} \ldots z_{m}^{\prime} z
$$

and the hypothesis is that

$$
u_{h}^{\prime} z_{1}^{\prime} \ldots z_{m}^{\prime} z \equiv u_{h}^{\prime \prime} z_{1}^{\prime \prime} \ldots z_{n}^{\prime \prime} z \quad(\rho) .
$$

Suppose that the canonical word associated with $x(z \rho)$ is $u_{h}^{*} z_{1}^{*} \ldots z_{p^{*}}^{*}$ :

Define $i^{\prime}(r) \in I(r=1,2, \ldots, m)$ by $z_{r}^{\prime} \in S_{i \prime(r)}$. Similarly define $i^{\prime \prime}(r)(r=1,2, \ldots, n)$ and $i^{*}(r)(r=1,2, \ldots, p)$ by $z_{r}^{\prime \prime} \in S_{i^{\prime \prime}(r)}$, and $z_{r}^{*} \in S_{i^{*}(r)}$.

Suppose that $z \in S_{j}$. Several cases can arise.

(a) $i^{\prime}(m) \neq j ; \quad i^{\prime \prime}(n) \neq j$.

(b) $i^{\prime}(m)=j$, and $z_{m}^{\prime} z \notin U \phi_{j} ; \quad i^{\prime \prime}(n) \neq j$.

(c) $i^{\prime}(m)=j$, and $z_{m}^{\prime} z \in U \phi_{j} ; \quad i^{\prime \prime}(n) \neq j$.

(d) $i^{\prime}(m)=j$, and $z_{m}^{\prime} z \notin U \phi_{j} ; \quad i^{\prime \prime}(n)=j$, and $z_{n}^{\prime \prime} z \notin U \phi_{j}$.

(e) $i^{\prime}(m)=j$, and $z_{m}^{\prime} z \in U \phi_{j} ; \quad i^{\prime \prime}(n)=j, \quad$ and $z_{n}^{\prime \prime} z \notin U \phi_{j}$.

$(f) i^{\prime}(m)=j$, and $z_{m}^{\prime} z \in U \phi_{j} ; \quad i^{\prime \prime}(n)=j$, and $z_{n}^{\prime \prime} z \in U \phi_{j}$.

Other cases are not essentially different.

In case (a), the two words (3) and (4) are canonical words and, being equivalent under $\rho$, they are therefore identical. It follows that $x=y$.

In case $(b)$, the word (4) is in canonical form, and is therefore identical to $u_{h}^{*} z_{1}^{*} \ldots z_{p}^{*}$. In particular

$$
z_{p}^{*}=z
$$

The word (3) is, however, not canonical, since $z_{m}^{\prime}$ and $z$ are both in $S_{j}$. If we apply to it the standard process (see [6]) for reducing a word to canonical form, the reduction begins

$$
z_{m}^{\prime} z=u_{j}^{(1)} z^{\prime}
$$

where $z^{\prime}$ is a non-trivial coset representative in $S_{j}$, and is the final coset representative in the canonical word associated with (3), i.e. in the word $u_{h}^{*} z_{1}^{*} \ldots z_{p}^{*}$. Thus $z^{\prime}=z_{p}^{*}$, and combining this with (5), we get $z^{\prime}=z$. Thus (6) becomes $z_{m}^{\prime} z=u_{j}^{(1)} z$ and, since $S_{j}$ is cancellative, this gives $z_{m}^{\prime}=u_{j}^{(1)}$, which contradicts the assumption that $z_{m}^{\prime}$ is a non-trivial coset representative. Thus case $(b)$ cannot arise.

In case $(c)$, the word (4) is again in canonical form, so that $z_{p}^{*}=z$. The standard reductior of (3) to canonical form begins

$$
z_{m}^{\prime} z=u_{j}^{(1)}
$$

Then $u_{j}^{(1)}$ changes to $u_{i^{\prime}(m-1)}^{(1)}$, and the reduction continues

$$
z_{m-1}^{\prime} u_{i^{\prime}(m-1)}^{(1)}=u_{i^{\prime}(m-1)}^{(2)} z^{\prime},
$$

where $z^{\prime}$ is a non-trivial coset representative in $S_{i^{\prime}(m-1)}$, and is the final coset representative in the canonical word associated with (3). Thus $z^{\prime}=z_{p}^{*}=z$. It follows therefore that $z^{\prime} \in S_{j}$, and from (7) we then conclude that $i^{\prime}(m-1)=j$. Hence $i^{\prime}(m-1)=i^{\prime}(m)$, which contradicts the assumption that $u_{h}^{\prime} z_{1}^{\prime} \ldots z_{m}^{\prime}$ is a canonical word. Thus case $(c)$ cannot arise. 
In case $(d)$, neither (3) nor (4) is a canonical word. The reduction of (3) to canonical form must begin

$$
\begin{aligned}
z_{m}^{\prime} z & =u^{(1)} z_{p}^{*}, \\
z_{m-1}^{\prime} u^{(1)} & =u^{(2)} z_{p-1}^{*},
\end{aligned}
$$

(leaving out the suffixes of the $u$ 's, as we may without confusion). The reduction of (4) begins

From (8) and (10),

$$
\begin{aligned}
z_{n}^{\prime \prime} z & =v^{(1)} z_{p}^{*}, \\
z_{n-1}^{\prime \prime} v^{(1)} & =v^{(2)} z_{p-1}^{*} .
\end{aligned}
$$

and therefore, by cancellation in $S_{j}$,

$$
\left(u^{(1)}\right)^{-1} z_{m}^{\prime}=\left(v^{(1)}\right)^{-1} z_{n}^{\prime \prime} .
$$

But this, by the uniqueness of the coset representation (2), implies that $u^{(1)}=v^{(1)}$ and $z_{m}^{\prime}=z_{n}^{\prime \prime}$. In the same way, from (9) and (11) we can now conclude that $u^{(2)}=v^{(2)}$ and $z_{m-1}^{\prime}=z_{n-1}^{\prime \prime}$. This may be continued. Now it is clear that the canonical word associated with (3) must contain precisely $m$ coset representatives. Similarly the canonical word associated with (4) contains $n$ coset representatives. Hence $m=n=p$, and we can continue the above argument, proving that $z_{r}^{\prime}=z_{r}^{\prime \prime}(r=1, \ldots, m)$. In the final stage of the simultaneous reduction of (3) and (4) to canonical form we get

$$
\begin{aligned}
u^{\prime} u^{(n)} & =u^{*} \\
u^{\prime \prime} u^{(n)} & =u^{*}
\end{aligned}
$$

from which we immediately conclude that $u^{\prime}=u^{\prime \prime}$. We have thus proved that the canonical words associated with $x$ and $y$ are identical. That is, we have proved that $x=y$.

In case $(e)$, the reductions begin

and

$$
\begin{aligned}
z_{m}^{\prime} z & =u^{(1)}, \\
z_{m-1}^{\prime} u^{(1)} & =u^{(2)} z_{p}^{*}, \\
z_{n}^{\prime \prime} z & =v^{(1)} z_{p}^{*} .
\end{aligned}
$$

It follows that $z_{p}^{*} \in S_{j}$, and therefore $z_{m-1}^{\prime}$ also belongs to $S_{j}$. Hence $i^{\prime}(m-1)=i^{\prime}(m)$, which contradicts the assumption that $u_{h}^{\prime} z_{1}^{\prime} \ldots z_{m}^{\prime}$ is a canonical word. Thus case $(e)$ cannot arise.

In case $(f)$, the reductions begin

and

$$
\begin{aligned}
z_{m}^{\prime} z & =u_{j}^{(1)}, \\
z_{m-1}^{\prime} u_{k}^{(1)} & =u_{k}^{(2)} z_{p}^{*}, \\
z_{n}^{\prime \prime} z & =v_{j}^{(1)} \\
z_{n-1}^{\prime \prime} v_{k}^{(1)} & =v_{k}^{(2)} z_{p}^{*} .
\end{aligned}
$$

(We write $k$ for $i^{\prime}(m-1)$ and $i^{\prime \prime}(n-1)$; it is obvious that these are equal, since both are equal to $i^{\bullet}(p)$.) It follows from (12) and (14) that

$$
e_{j}=\left(u_{j}^{(1)}\right)^{-1} z_{m}^{\prime} z=\left(v_{j}^{(1)}\right)^{-1} z_{n}^{\prime \prime} z
$$

and therefore, by cancellation in $S_{j},\left(u_{j}^{(1)}\right)^{-1} z_{m}^{\prime}=\left(v_{j}^{(1)}\right)^{-1} z_{n}^{\prime \prime}$. By the uniqueness of the coset representation (2), this implies that $u_{j}^{(1)}=v_{j}^{(1)}$ and $z_{m}^{\prime}=z_{n}^{\prime \prime}$. From (13) and (15) we now get 
$z_{p}^{*}=\left(u_{k}^{(2)}\right)^{-1} z_{m-1}^{\prime} u_{k}^{(1)}=\left(v_{k}^{(2)}\right)^{-1} z_{n-1}^{\prime \prime} v_{k}^{(1)}$, and since $u_{k}^{(1)}=v_{k}^{(1)}$ we can use cancellation in $S_{k}$ to obtain $\left(u_{k}^{(2)}\right)^{2-1} z_{m-1}^{\prime}=\left(v_{k}^{(2)}\right)^{-1} z_{n-1}^{\prime \prime}$. From this it follows that $u_{k}^{(2)}=v_{k}^{(2)}$ and $z_{m-1}^{\prime}=z_{n-1}^{\prime \prime}$. This argument may now be continued exactly as in case $(d)$, giving $x=y$.

We have proved that $\Pi_{U}^{*} S_{i}$ is right-cancellative. By taking the elements of $\Pi_{U}^{*} S_{i}$ in a canonical form $z_{1} \ldots z_{n} u$, with the $z$ 's as left-coset representatives, and going through a procedure exactly dual to the above, we can show that $\Pi_{U}^{*} S_{i}$ is also left-cancellative.

This completes the proof of Theorem 2.1.

3. A counterexample. First, we recall some definitions and basic results. A relation $\rho$ on a semigroup $S$ may be thought of as a subset of $S \times S$. If $\rho$ is an equivalence relation, the statement $(x, y) \in \rho$ will usually be written $x \equiv y(\rho)$. The congruence relation generated by a set $\Sigma$ of elements of $S \times S$ is defined as the smallest congruence on $S$ containing $\Sigma$.

We define a free semigroup as the set of finite and non-empty words in a set of generators, multiplication being defined by juxtaposition. The following result is well-known [1].

For every semigroup $S$ there exists a free semigroup $F$ (not unique), and a congruence $\sigma$ on $F$ such that $F / \sigma \simeq S$.

This may be interpreted as giving the presentation of $S$ by generators and defining relations. The generators are the generating set $\Gamma$ of the free semigroup $F$, and the defining relations are any collection of elements of $F \times F$ which generate the congruence $\sigma$.

The manner in which a set of defining relations $\Sigma \subseteq F \times F$ generate a congruence $\sigma$ on the free semigroup $F$ is easy to describe. Suppose that $f=p s q, g=p t q$, where $p, q \in F$ or are empty, and $(s, t) \in \Sigma$. Then we shall say that each of $f$ and $g$ is obtained from the other by an elementary transformation based on the defining relation $(s, t)$. For two elements $x, y$ of $F$, we then have that $x \equiv y(\sigma)$ if and only if $y$ can be obtained from $x$ by a finite (perhaps empty) sequence of elementary transformations each of which is based on a defining relation in $\Sigma$.

The following notational device will be useful. If $w$ (say) denotes an element of the free semigroup $F$, then the element $w \sigma$ of the semigroup $F / \sigma$ will be denoted by $w$. That is to say, if a word in the generators of a semigroup is denoted by a certain letter, then the same letter in bold face is used to denote the corresponding element of the semigroup.

In what follows it will be unnecessary to use the precise notation of $\$ 1$, and we shall usually make no distinction between a semigroup and its isomorphic image.

Our assertion is that there exist cancellative semigroups $S$ and $T$, each of which contains a cancellative semigroup $U$ as a unitary subsemigroup, for which the cancellative embedding $[S, T ; U]$ is not possible. This is shown by the following example.

Let $U$ be $\{u, v, x, y\}$, a free semigroup on four generators. Let $S$ have generators $\{u, v, x, y, a, b\}$ and defining relations $\{(a u, b v),(a x, b y)\}$. Let $T$ have generators $\{u, v, x, y, c, d, f\}$ and defining relations $\{(u c, x d),(y d, v f)\}$. Then

(i) $U$, being free, is a cancellative semigroup [7];

(ii) $S$ is cancellative, and $U$ is a unitary subsemigroup of $S$;

(iii) $T$ is cancellative, $U$ is a unitary subsemigroup of $T$, and $\mathbf{c} \neq \mathbf{f}$;

(iv) the cancellative embedding $[S, T ; U]$ is not possible.

Assume for the moment that (ii) and (iii) are true, and suppose that (iv) is not true. That 
is, suppose that there exists a cancellative semigroup $K$ containing $S$ and $T$ as subsemigroups, with $S \cap T=U$. Then in $K$,

$$
\text { auc }=\text { axd }=\text { byd }=\text { bvf }=\text { auf, }
$$

and therefore, by cancellation, $\mathbf{c}=\mathbf{f}$. But $\mathbf{c} \neq \mathbf{f}$ in $T$. Thus we have a contradiction, and the cancellative embedding $[S, T ; U]$ is not possible.

It remains to prove (ii) and (iii) above. It is easy to show that the subsemigroup of $S$ generated by $u, v, x, y$ is free, so that (up to isomorphism) $U$ is a subsemigroup of $S$. Now let $u^{(1)}, u^{(2)}$ be words in the generators of $U$, and $s$ a word in the generators of $S$, and suppose that $\mathbf{u}^{(1)} \mathbf{s}=\mathbf{u}^{(2)}$. Then $u^{(2)}$ and $u^{(1)} s$ are connected by a sequence (perhaps empty) of elementary transformations based on the defining relations of $S$. Now it is clear that the word $u^{(2)}$ cannot be changed by an elementary transformation based on a definining relation of $S$, since neither of the defining relations of $S$ involves a word entirely in the generators of $U$. Thus the sequence connecting $u^{(2)}$ and $u^{(1)} s$ must be empty. That is to say, the two words must be identical. In particular, $s$ must be a word in the generators of $U$. We have thus proved that $\mathbf{u}^{(1)} \mathbf{s} \in U \Rightarrow \mathbf{s} \in U$, and the dual result may be proved similarly, showing that $U$ is a unitary subsemigroup of $S$.

Next, we show that $S$ is left-cancellative. The following lemma clearly establishes this.

Lemma 3.1. Let $g$ be a generator of $S$, and let $s^{(1)}, s^{(2)}$ be words in the generators of $S$. Suppose that $g s^{(1)}$ and $g s^{(2)}$ are connected by a sequence $\mathscr{S}_{1}$ of elementary transformations based on the defining relations of $S$. Then there is a sequence $\mathscr{S}_{2}$ of elementary transformations connecting $s^{(1)}$ and $s^{(2)}$, and the length of $\mathscr{S}_{2}$ is not greater than the length of $\mathscr{S}_{1}$.

We prove this lemma by induction on the length $l$ of $\mathscr{S}_{1}$. A simple verification shows that it holds for $l=1$. Suppose therefore that it holds for $l<n$, and that $g s^{(1)}$ and $g s^{(2)}$ are connected by a sequence of elementary transformations of length $n(\geqslant 2)$. We can clearly restrict ourselves to the case where the first step is $g s^{(1)} \rightarrow s^{(3)}$ and the last step is $s^{(4)} \rightarrow g s^{(2)}$, where neither $s^{(3)}$ nor $s^{(4)}$ begins with $g$, for otherwise the induction hypothesis would immediately give the desired conclusion. Thus $g$ must be the first letter in a word occurring in a defining relation of $S$; in other words $g$ must be $a$ or $b$. It will be sufficient to consider the case where $g=a$, and where the first step is based on the relation $(a u, b v)$. That is,

$$
g s^{(1)}=a u s^{(s)} \text { and } s^{(3)}=b v s^{(s)} .
$$

At the end of the sequence there are two possibilities. Either

or

(a) $g s^{(2)}=a u s^{(6)}$ and $s^{(4)}=b v s^{(6)}$;

(b) $g s^{(2)}=a x s^{(6)}$ and $s^{(4)}=b y s^{(6)}$.

In case $(a)$ we have a sequence of $n-2$ elementary transformations connecting $b v s^{(5)}$ and $b v s^{(6)}$. Therefore by the induction hypothesis there is a sequence of at most $n-2$ steps connecting $v s^{(5)}$ and $v s^{(6)}$. Applying the induction hypothesis again, we find that $s^{(5)}$ and $s^{(6)}$ are connected by a sequence of elementary transformations, and the length of this sequence is at most $n-2$. Now $s^{(1)}=u s^{(5)}$ and $s^{(2)}=u s^{(6)}$, so that obviously there is a sequence of at most $n-2$ elementary transformations connecting $s^{(1)}$ and $s^{(2)}$.

In case $(b)$ we have a sequence of $n-2$ elementary transformations connecting $b v s^{(5)}$ and $b y s^{(6)}$. By induction therefore, $v s^{(5)}$ and $y s^{(6)}$ are connected by a sequence of elementary 
transformations. But this is impossible, since no elementary transformation based on a defining relation of $S$-and hence no finite sequence of such transformations-can change a word beginning with $v$ to a word not beginning with $v$. Therefore case $(b)$ cannot arise, and we have proved that $S$ is left-cancellative.

The proof that $S$ is right-cancellative is closely similar. By the same inductive method as above we can prove a lemma differing in statement from the lemma just proved only in that $s^{(1)} g$ and $s^{(2)} g$ replace $g s^{(1)}$ and $g s^{(2)}$. Thus $S$ is a cancellative semigroup.

A similar approach is used to prove that $U$ is a unitary subsemigroup of $T$, and that $T$ is cancellative. The proof involves no new ideas and is therefore omitted. To prove that $\mathbf{c} \neq \mathbf{f}$ in $T$, consider the class of words in the generators of $T$ which can be obtained from the word $c$ by means of a finite sequence of elementary transformations based on the defining relations of $T$. It is immediately obvious that this class consists of the single word $c$. It certainly does not contain the word $f$, and therefore the two elements $\mathrm{c}$ and $\mathrm{f}$ of $T$ are distinct.

\section{REFERENCES}

1. G. Birkhoff, Lattice theory (Foreword on Algebra), Revised Edition, Amer. Math. Soc. Colloquium publications XXV (New York, 1948).

2. P. Dubreil, Contribution à la théorie des demigroupes, Mem. Acad. Sci. Inst. France 63 (1941), no. 3, 1-52.

3. M. Hall, The theory of groups, (New York, 1959).

4. J. M. Howie, Embedding theorems with amalgamation for semigroups, Proc. London Math. Soc. (3) 12 (1962), 511-534.

5. J. M. Howie, Some problems in the theory of semigroups, Thesis, Oxford (1961).

6. A. G. Kurosh, The theory of groups, vol. II, (New York, 1955), 29-32.

7. F. W. Levi, On semigroups, Bull. Calcutta Math. Soc. 36 (1944), 141-146.

\section{THE UNIVRSITY}

\section{Glasgow}

